\title{
Development of Highly Sensitive Metal-Free Tetraphenylporphyrin- Based Optical Oxygen Sensing Materials along with ILs and AgNPs
}

\author{
Merve Zeyrek Ongun* \\ Chemistry Technology Program, Izmir Vocational High School, University of Dokuz Eylul, Izmir, Turkey \\ *merve.zeyrek@deu.edu.tr
}

Received: 03 January 2019

Accepted: 12 March 2019

DOI: $10.18466 /$ cbayarfbe. 507626

\begin{abstract}
An oxygen sensitive optical chemical sensor has been developed based on fluorescence quenching of the meso-tetraphenylporphyrin $\left(\mathrm{H}_{2} \mathrm{TPP}\right)$ immobilized in a silicone derivative along with silver nanoparticles (AgNPs) and different ionic liquids (ILs). Emission spectra of the $\mathrm{H}_{2}$ TTP doped thin film exhibited an increment due to the formation of an associated complex between $\mathrm{H}_{2}$ TPP and AgNPs. The offered thin films responded to the oxygen in the direction of quenching with extreme sensitivity. Emission and decaytime measurements of the $\mathrm{H}_{2}$ TPP in thin solid matrices were studied in the concentration range of $0-100 \%$ $\mathrm{p}\left(\mathrm{O}_{2}\right)$. Utilization of the porphyrin dye along with AgNPs and ionic liquid as an additive exhibited higher oxygen sensitivity with respect to the additive-free forms and resulted in many advances such as linear response, improvement in sensor dynamics and extreme sensitivity. Together with additives, the mesotetraphenylporphyrin-based composites yielded higher Stern-Volmer constant (Ksv), faster response time, and larger linear response range when compared with the additive-free form. The response time of the sensor has been recorded as $90 \mathrm{~s}$.
\end{abstract}

Keywords: meso-tetraphenylporphyrin $\left(\mathrm{H}_{2} \mathrm{TPP}\right)$, silver nanoparticles, ionic liquids, optical oxygen sensing.

\section{Introduction}

Recently, the luminescence technique is preferred as a convenient optical chemical sensing approach due to its sensitivity and selectivity. Fluorescence-based sensing materials are used for the analysis of many different inorganic and organic structures and the response is mainly based on fluorescence quenching [1]. Last decades, tetraphenylporphyrins (TPPs) become in the limelight for researchers that are used for a variety of applications and devices, such as optoelectronic, luminescent and molecular logic devices [2], supramolecular self-assembly [3] and solar energy harvesting systems [4]. Metal-free and metal bearing forms of the TPPs are unique objects for $\mathrm{O}_{2}$ sensing applications. Fluorescence response of the tetraphenylporphyrins is efficiently quenched by triplet oxygen; nonetheless whether in solution or in the polymeric matrices in the embedded form [5].

The meso-tetraphenylporphyrins $\left(\mathrm{H}_{2} \mathrm{TPP}\right)$ have been studied many times from the point of synthesis and applications for few decades. They can be utilized both in sensor applications as thin films or nanofibers together with fiber-optics that enable oxygen sensing [6]. The TPP derivative-based thin film owns medium to high photostability depending on the chosen polymeric matrix material. Topal et. al. reported high sensitivity towards oxygen $\left(\mathrm{I}_{0} / \mathrm{I}=5.02\right)$ when used the $\mathrm{H}_{2} \mathrm{TPP}$ dye in poly(1-trimethylsilyl-1-propyne) for a large concentration range [7].
In the novel studies, researchers work on the enhancement of the stability and $\mathrm{O}_{2}$ sensing characteristics of porphyrin dyes in nano and microscale solid matrices. The sensing properties of fluorescent sensors sorely attach to the kind of polymeric material and on their eventual modifications.

Recently, the researchers put forth that the addition of nano/micro-chemistry reagents such as AgNPs [8], AuNPs [9] and other additives like ionic liquids [10] bring superior features in the design of the sensor.

In this paper, oxygen sensing composite relaying on the reversible fluorescence quenching of $\mathrm{H}_{2} \mathrm{TPP}$ by the triplet oxygen is described. Initial signal intensity and consequently sensitivity of the $\mathrm{H}_{2} \mathrm{TPP}$ dye exhibited an enhancement due to the utilized additives. Herein we mainly focused on the clarification of the mechanism behind the sensor, the response characteristics, and the stability. The oxygen sensing behavior of the mesotetraphenylporphyrin $\left(\mathrm{H}_{2} \mathrm{TPP}\right)$ was investigated in the poly(1-trimethylsilyl-1-propyne) matrix in the solid state in form of thin film in presence of the silver nanoparticles (AgNPs), perfluorochemical (PFC) and the various ionic liquids (ILs). The additives; ILs and AgNPs enhanced the sensor dynamics including susceptibility, durability, and stability of the porphyrin. The sensitivity of the $\mathrm{H}_{2}$ TPP towards $\mathrm{O}_{2}$ gas was tested with fluorescence emission measurements as well as decay time results. As far as we know, the $\mathrm{H}_{2} \mathrm{TPP}$ was 
used for the first time along with AgNPs and ILs in poly (TMSP)-based thin film as an oxygen sensing slide.

\section{Materials and Methods 2.1. Materials}

The meso-Tetraphenylporphyrin $\left(\mathrm{H}_{2} \mathrm{TPP}\right)$ dye and perfluorododecaonic acid (PFC) were purchased from Sigma-Aldrich. Polymeric support material; Poly (1trimethylsilyl-1-propyne) was from ABCR Company. Room temperature ionic liquids (RTILs), 1-ethyl-3methylimidazolium tetra fluoroborate $\left(\left[\mathrm{EMIM}^{+}\right]\left[\mathrm{BF}_{4}^{-}\right]\right)$ (IL-I), 1-butyl-3-methyl imidazolium tetrafluoroborate; $\left(\left[\mathrm{BMIM}^{+}\right]\left[\mathrm{BF}_{4}^{-}\right]\right) \quad$ (IL-II) and 1-butyl-3-methyl imidazolium thiocyanate $\left(\left[\mathrm{BMIM}^{+}\right]\left[\mathrm{SCN}^{-}\right]\right)$(IL-III) were provided from Fluka and Sigma-Aldrich, respectively. Sodium borohydride $\left(\mathrm{NaBH}_{4}\right)$ and silver nitrate $\left(\mathrm{AgNO}_{3}\right)$ were of $99 \%$ purity and provided from Sigma-Aldrich. $\mathrm{O}_{2}$ and $\mathrm{N}_{2}$ gas-filled tube were of 99.99\% purity and derived Gunes Company, Izmir, Turkey. Fig. 1. reveals schematic design of the utilized metal-free porphyrin dye and imidazolium salts, respectively.

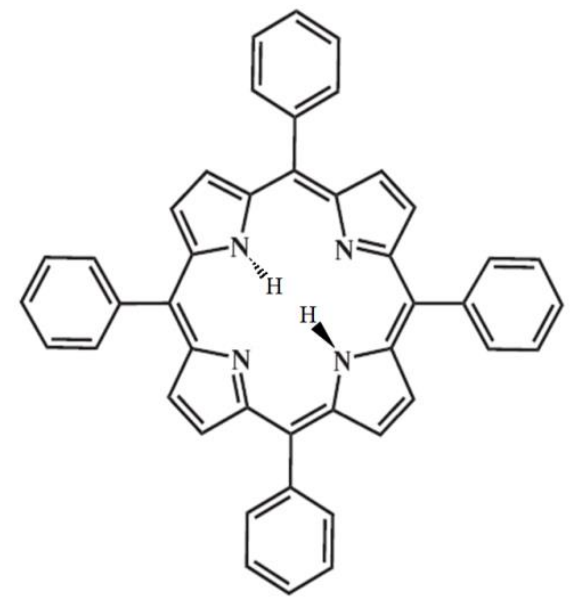

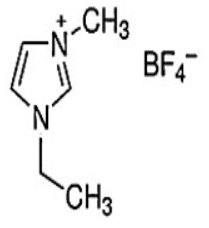
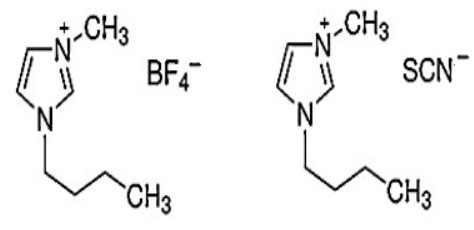

IL - I

IL - II

IL - III
Figure 1. The metal-free meso-tetraphenylporphyrin dye and imidazolium salts: IL-I: 1-ethyl-3methyl imidazolium tetrafluoroborate, IL-II: 1-butyl-3-methyl imidazolium tetrafluoroborate, IL-III: 1-butyl-3methylimidazolium thiocyanate.

\subsection{Instrumentation}

The emission based fluorescence and decay time measurements were implemented by a spectrofluorometer of Edinburg Instruments; FLS920. The sensing agents were excited with a xenon lamp during intensity based measurements. The emission slits were adjusted to $5 \mathrm{~nm}$. The Instrument Response
Function (IRF) of the instrument was recorded using a non-fluorescent of LUDOX (30\%, Sigma Aldrich) in water. The solution was considered to be wavelength independent and hold in a conventional quartz cell during IRF measurements.

The decay times were calculated by reiterative convolution with a weighted, nonlinear least-squares approach utilizing the measured IRF and decay time data under the same conditions. The chi-square values $(\chi 2)$ were kept less than 1.3 and plots of weighted residuals were used to evaluate the fit between the calculated and measured decays. A zeta sizer instrument (Malvern Instruments Ltd.), was used for the size dispersion tests of the synthesized metallic AgNPs in dispersed form in water.

\section{Results and Discussion \\ 3.1. Oxygen sensing measurements}

Certain concentrations of the oxygen and nitrogen gases were prepared in a gas mixing system (Sonimix 7000A) for the concentration range of $0.0-100.0 \%$ The flow velocity of the gas mixtures was adjusted at $<500$ $\mathrm{mL} / \mathrm{min}$. The blended gases were inserted into the oxygen sensing matrices by a steel diffuser needle. The emission spectra of the sensing slides were registered under a continuous flow of $\mathrm{O}_{2}$ in the partial pressure range of 1.01-101.32 $\mathrm{kPa}$.

\subsection{Preparation of silver nanoparticles (AgNPs)}

Metallic AgNPs were synthesized by using Solomon's method [11]. $30 \mathrm{~mL}$ of $1.0 \mathrm{mM}$ of $\mathrm{AgNO}_{3}$ was added dropwise into the $90 \mathrm{~mL}$ of $2.0 \mathrm{mM} \mathrm{NaBH}_{4}$ solution in an ice bath under continuous magnetic stirring. An excess amount of sodium borohydride was used for the complete reduction of the silver ions and meanwhile, to prevent the coagulation of the AgNPs. The silver nanoparticles corresponding to the average particle size of $80 \mathrm{~nm}$ were synthesized (See Fig. 2). Separation of the silver nanoparticles in dispersed water was performed by centrifugation. Following the separation by centrifugation, we applied a few consecutive washing processes with distilled water and THF.

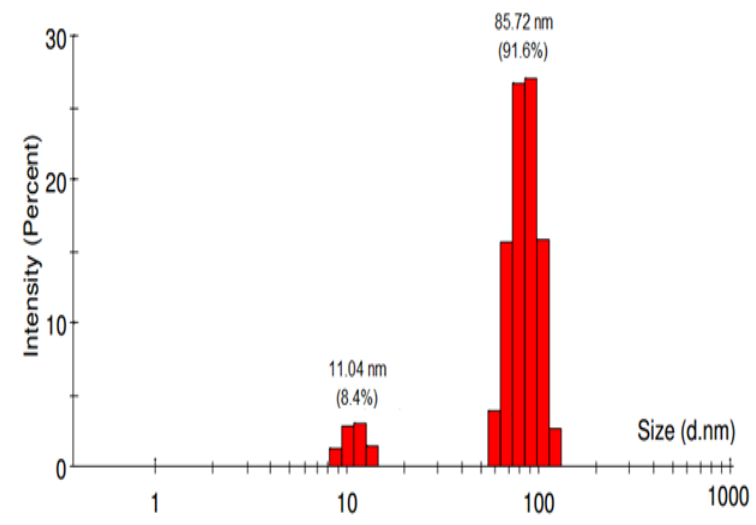

Figure 2. Size distribution test results of silver nanoparticles. 


\subsection{Preparation of oxygen sensing solid matrix}

In this work, the polymeric thin films were prepared by mixing $75 \mathrm{mg}$ of poly(TMSP), $0.25 \mathrm{mg}$ porphyrin dye and $36 \mathrm{mg}$ of different kinds of ILs in $2.5 \mathrm{~mL}$ of toluene.

On the other hand, the ILs and AgNPs ( $80 \mathrm{~nm})$ were added into the matrix in order to control the effective coupling distance and to improve the scattering/absorption ratio. The viscous composites were spread onto a polyester (Mylar TM type) support using knife coating method. Tencor Alpha Step 500 Prophylometer was used to measure thicknesses of the coated thin films and it found to be $7.48 \pm 0.13 \mu \mathrm{m}(\mathrm{n}=8)$. Each thin film was cut to $11 \times 30 \mathrm{~mm}$ size, placed in the cuvette and the fluorescence-based measurements were recorded.

\subsection{Spectral characterization of the $\mathrm{H}_{2} \mathrm{TPP}$ dye}

Absorption spectrum of $\mathrm{H}_{2} \mathrm{TPP}$ was recorded in toluene and poly(TMSP) without any additive. Spectral information regarding to the $\mathrm{H}_{2}$ TPP was summarized in Table 1. The $\mathrm{H}_{2}$ TTP exhibited a typical strong Soret absorption band and four weak $Q$ bands in the absorption spectra in toluene. When incorporated in poly(TMSP), in the form of thin films, exhibited small blue shifts extending to $8 \mathrm{~nm}$, with respect to the toluene (See Fig. S1).

Table 1. Absorption-based data of the $\mathrm{H}_{2}$ TPP in $5 \times 10^{-6}$ $\mathrm{M}$ toluene and in the solid matrix.

\begin{tabular}{llccccc}
\hline Dye & Matrix & $\begin{array}{c}\text { B } \\
\text { band }\end{array}$ & \multicolumn{3}{c}{ Q- bands $(\boldsymbol{\lambda} / \mathbf{n m})$} \\
\hline & & & Qy & Qy & Qx & Qx \\
& & & $(1.0)$ & $(1.0)$ & $(1.0)$ & $(0.0)$ \\
\cline { 4 - 7 } & Toluene & 422 & 516 & 549 & 592 & 655 \\
$\mathrm{H}_{2}$ TPP & Thin & & & & & \\
& Film & 418 & 514 & 548 & 591 & 647 \\
& & & & & & \\
\end{tabular}

$\mathrm{H}_{2}$ TPP exhibited strong double phosphorescence emission band in around near infrared region (NIR) in toluene and poly(TMSP) based composite when excited at $418 \mathrm{~nm}$. Thee $\mathrm{H}_{2}$ TPP dye showed the emission peaks ( $\mathrm{Qx}(0,0)$ and $\mathrm{Qx}(0,1))$ which can be appeared at $\sim 650$ and $\sim 720 \mathrm{~nm}$ in the absence of the oxygen gas (See Table 2).

\subsection{Effect of PFC on oxygen sensitivity}

As far as we know, utilization of the perfluorochemicals and oxygen sensitive dye embedded in polymeric matrix presents an improvement in the sensing ability compared with the condition of the absence of PFC [7].
Table 2. Emission spectra and oxygen sensitivity related data of $\mathrm{H}_{2} \mathrm{TPP}$ in $5 \times 10^{-6} \mathrm{M}$ toluene and poly(TMSP)-thin film in the absence of the PFC.

\begin{tabular}{lcccc}
\hline Compound & Matrix & $\begin{array}{c}\lambda_{\text {em }} \\
\mathbf{Q x} \\
(\mathbf{0 . 0})\end{array}$ & $\begin{array}{c}\lambda_{\text {em }} \\
\mathbf{Q x} \\
(\mathbf{0 . 1})\end{array}$ & $\begin{array}{c}\mathbf{I}_{\mathbf{0}} / \mathbf{I}_{10} \\
{ }_{\mathbf{0}} \mathbf{Q x} \\
(\mathbf{0 . 1})\end{array}$ \\
& & & & \\
\hline & Toluene & 651 & 717 & 2.20 \\
$\mathrm{H}_{2} \mathrm{TPP}$ & & & & \\
& $\begin{array}{l}\text { Thin } \\
\text { Film }\end{array}$ & 652 & 717 & 1.46 \\
& & & & \\
\hline
\end{tabular}

Oxygen exhibits an extraordinary solubility in perfluoro compounds. In other terms, the oxygen molecules are able to employ the molecular cavities within PFCs without any chemical reactions or interactions [12]. Therefore, the PFC was embedded into the solid matrix to provide better solubility and enhanced diffusion of the oxygen.

In our previous studies, we optimized the concentration of the PFC in the poly(TMSP) based composites [13]. Optimal quantity of the PFC in the cocktail composition was investigated by different amounts of the PFC in the polymeric matrix. In order to understand the effect of the presence of the PFC on the oxygen sensitivity, the absorption and emission spectra of the $\mathrm{H}_{2} \mathrm{TPP}$ dye was measured for PFC-free, 0.5, 1.0 and $2.0 \mathrm{mg}$ PFC containing composites, respectively (See Fig. S 2-4). The best sensitivity was obtained when $2.0 \mathrm{mg}$ of PFC was utilized in the matrix [13].

\subsection{Effect of ILs on oxygen sensing}

Herein, we used the ionic liquids consisting of imidazolium ring with different anionic parts; $\mathrm{BF}_{4}{ }^{-}$and $\mathrm{CN}^{-}$. We encoded the ionic liquids of $\left[\mathrm{EMIM}^{+}\right]\left[\mathrm{BF}_{4}^{-}\right]$, $\left[\mathrm{BMIM}^{+}\right]\left[\mathrm{BF}_{4}^{-}\right]$and $[\mathrm{BMIM}]\left[\mathrm{SCN}^{-}\right]$as IL-I, IL-II and IL-III, respectively. The Stern-Volmer constants $\left(\mathrm{K}_{\mathrm{SV}}\right)$ and the $\mathrm{I}_{0} / \mathrm{I}_{100}$ ratio of the $\mathrm{H}_{2} \mathrm{TPP}$ dye in EC were shown in the Table 3.

We obtained the highest $\mathrm{Ksv}$ and $\mathrm{I}_{0} / \mathrm{I}_{100}$ ratio when we used the $\left[\mathrm{BMIM}^{+}\right]\left[\mathrm{BF}_{4}^{-}\right]$as the additive. Oxygen induced response of the $\left[\mathrm{EMIM}^{+}\right]\left[\mathrm{BF}_{4}^{-}\right]$was better with respect to the $[\mathrm{BMIM}]\left[\mathrm{SCN}^{-}\right]$which can be attributed to the presence of the fluorine atoms. According to the previous work, 10 to 20 times higher oxygen solubilities were reported in ILs bearing fluorine atoms than that of the observed in the aqueous phases or in the polymeric matrices $[14,15]$.

On the other hand, the length of the alkyl chain on the imidazolium part is also important in the interaction of the additive with the matrix. Therefore, the $\left[\mathrm{BMIM}^{+}\right]\left[\mathrm{BF}_{4}^{-}\right]$was also the best additive in terms of compatibility within the matrix. 
3.7. Fluorescence-based measurements of $\mathrm{H}_{2}$ TPP dye in thin films

In this work, we exploited metal-free mesotetraphenylporphyrin dye together with the several of ionic liquids as the $\mathrm{O}_{2}$ sensing material. Emission-based spectra of the $\mathrm{H}_{2}$ TPP was monitored in toluene as well as in poly(TMSP)-based thin films containing different kinds of ILs and PFC, under different oxygen partial pressures (See Table 3 ). When the polymer embedded $\mathrm{H}_{2}$ TPP was excited at $420 \mathrm{~nm}$, yields two well-shaped emission bands at the red edge of the electromagnetic spectrum, at 652 and $717 \mathrm{~nm}$, respectively. When exposed to the increasing concentrations of the oxygen, the fluorescence intensities at 652 and $717 \mathrm{~nm}$ exhibited a decrease which can be used as the analytical signal. The $\mathrm{H}_{2}$ TPP-based cocktail; named as $\mathrm{C} 1$, and, IL-I, ILII and IL-III doped composites named as C2, C3 and $\mathrm{C} 4$, exhibited varying intensity drops under the oxygen atmosphere in the presence of the silver nanoparticles (See Table 3). In all cases, the emission intensities decreased with the increasing quencher amounts. However, the highest oxygen-induced relative signal change was observed for the cocktail; C3. Upon exposure to $100 \%$ of $\mathrm{pO}_{2}$, the relative signal change of the $\mathrm{C} 3$ was $88 \%$ (See Table 3 ).

Herein, triplet oxygen quenched fluorescence of the $\mathrm{H}_{2}$ TPP dye in its excited state via collisions. Collisional quenching, which is also known as dynamic quenching, causes a non-radiative energy transfer. The relative signal change arising from the dynamic quenching depends on the triplet oxygen amounts and the oxygen diffusivity of the utilized polymer due to the variations in the frequency of collisions. For a homogeneous environment, a plot of the ratio of $\mathrm{I}_{0} / I$ or $\tau_{0} / \tau$ versus [O $\left.\mathrm{O}_{2}\right]$ yields a straight line with an intercept at 1 and a slope of $K_{\mathrm{SV}}$, which is a measure of sensor sensitivity. The following Stern-Volmer equation reveals the relationship between the quencher concentration, the fluorescence lifetimes and intensities [16]:

$$
I_{0} / I=\tau_{0} / \tau=1+K_{S V}\left[O_{2}\right]=1+k q \tau_{0}\left[O_{2}\right]
$$

where $\mathrm{I}_{0}$ and $\mathrm{I}$ are the fluorescence intensities and $\tau_{0}$ and $\tau$ are the fluorescence decay times in the absence and presence of oxygen, respectively. $\mathrm{K}_{\mathrm{SV}}$ is the SternVolmer constant and $\mathrm{kq}$ is the quenching constant, which is related to the diffusion ability of oxygen through the matrix.

The Stern-Volmer constant of the C3 based sensing slide was 1.86 times higher with respect to the $\mathrm{K}_{\mathrm{SV}}$ value of the $\mathrm{C} 1$ based film. The enhancement in the diffusion ability of the oxygen and consequently in the sensitivity was an expected result for us in ionic liquid modified matrices. The fluorescence spectra of the thin films after exposure to the oxygen in the concentration range of $0-100 \% \mathrm{pO}_{2}$ were presented between Fig. 3-6. The relative signal changes of the composites of $\mathrm{C} 1, \mathrm{C} 2$, $\mathrm{C} 3$ and $\mathrm{C} 4$ were found to be 4.72, 6.28, 8.80 and 2.72, respectively (see Table 3 ).
The oxygen-induced emission spectra and related calibration plots described by the equations for all of the composites were shown between Fig. $3-6$. All of them yielded good $R^{2}$ values. The oxygen-induced relative signal change enhanced from $50 \%(\mathrm{C} 1)$ to $88 \%(\mathrm{C} 3)$ when the additives were utilized with respect to the additive-free formulations. As mentioned earlier, the Stern-Volmer constant $\left(\mathrm{K}_{\mathrm{SV}}\right)$ also quantifies the quenching efficiency of the sensor. The calculated $\mathrm{K}_{\mathrm{SV}}$ values for all of the thin films lied in the range of $1.05 \times 10^{-2}-7.43 \times 10^{-2}$. Among them the $\mathrm{C} 3$ exhibited the highest $\mathrm{K}_{\mathrm{SV}}$ value.

The ratio $I_{0} / I_{100}$ is also representative of the sensitivity of a sensor, where a higher value means higher sensitivity. According to the literature, the $\mathrm{I}_{0} / \mathrm{I}_{100}$ value of 8.80 indicates a good sensitivity [17] and makes the C3 composition a promising material for the development of near-infrared luminescence-based oxygen sensing device. From the Fig. 5 it can be concluded that the cocktail $\mathrm{C} 3$ exhibited higher relative signal change and $\mathrm{K}_{\mathrm{SV}}$ values, better oxygen gas sensitivity with respect to the other cocktails. The limit of detection (LOD) was calculated considering the $100 \% \mathrm{~N}_{2}$ gas moiety as the blank and calculating the mean and the standard deviation (SD) for the 20 replicate measurements.
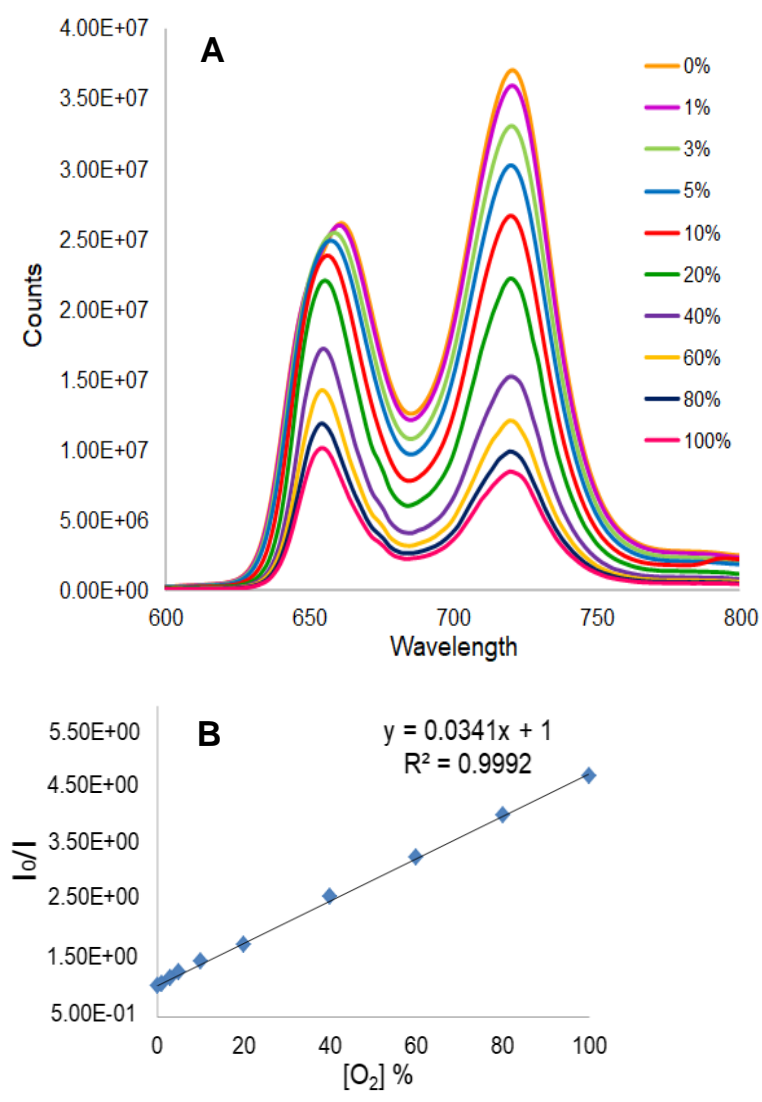

Figure 3. A: The emission spectra of free $\mathrm{H}_{2} \mathrm{TPP}$ dye doped in poly(TMSP) in the oxygen concentrations of 0-100\%. B: Stern-Volmer plot Io/I $=\mathrm{f}\left(\mathrm{pO}_{2}\right)$ of $\mathrm{H}_{2} \mathrm{TPP}$ for $\mathrm{O}_{2}$ sensing. 
Table 3. Emission based characteristics and oxygen sensitivity data of the $\mathrm{H}_{2} \mathrm{TPP}$ dye embedded in poly(TMSP)based thin films supported with different kinds of ILs in the presence of the PFC (2 mg) and AgNPs.

\begin{tabular}{|c|c|c|c|c|c|c|c|c|c|c|c|}
\hline Dye & Cocktail & $\begin{array}{c}\text { Ionic } \\
\text { Liquid }\end{array}$ & Additive & Medium & $\lambda_{\mathrm{ex}}$ & $\lambda_{e m}^{1}$ & $\lambda_{e m}^{2}$ & $\mathbf{I}_{0} / \mathbf{I}$ & $\begin{array}{c}\mathbf{K}_{\mathbf{S V}} \\
\left(\%^{-1}\right)\end{array}$ & $\mathbf{R}^{2}$ & LOD \\
\hline \multirow{4}{*}{$\mathrm{H}_{2} \mathrm{TPP}$} & $\mathrm{C} 1$ & - & PFC & Thin film & 420 & 652 & 717 & 4.72 & 0.0341 & 0.9994 & $1.32 \times 10^{-3}$ \\
\hline & $\mathrm{C} 2$ & IL-I & $\begin{array}{c}\text { AgNPs } \\
\text { PFC }\end{array}$ & Thin film & 420 & 656 & 720 & 6.28 & 0.0467 & 0.9935 & - \\
\hline & $\mathrm{C3}$ & IL-II & $\begin{array}{c}\text { AgNPs } \\
\text { PFC }\end{array}$ & Thin film & 420 & 652 & 716 & 8.80 & 0.0743 & 0.9918 & $4.12 \times 10^{-4}$ \\
\hline & $\mathrm{C} 4$ & IL-III & $\begin{array}{c}\text { AgNPs } \\
\text { PFC }\end{array}$ & Thin film & 420 & 658 & 720 & 2.72 & 0.0105 & 0.9181 & - \\
\hline
\end{tabular}
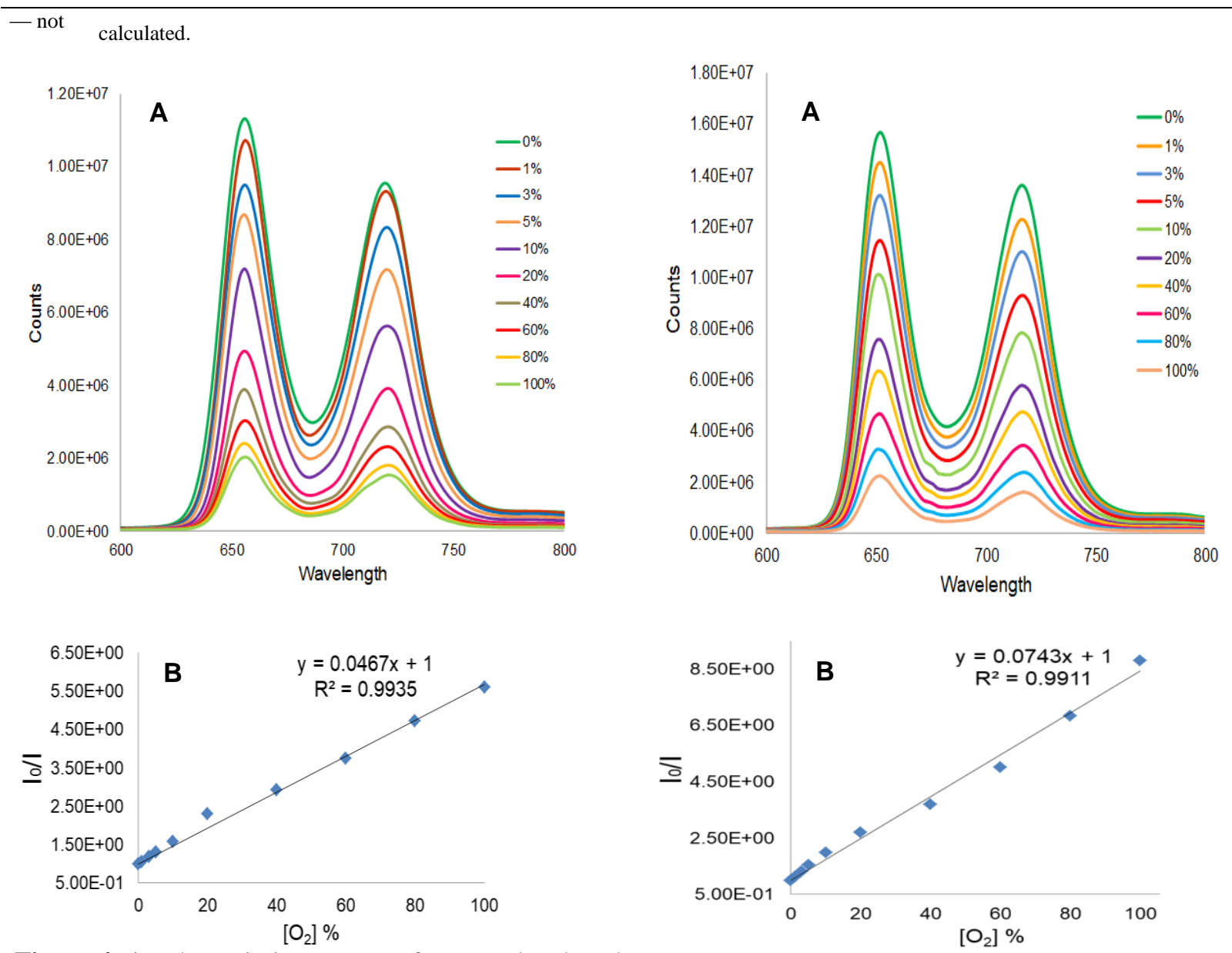

Figure 4. A: The emission spectra of $\mathrm{H}_{2}$ TPP dye doped in poly(TMSP) in the presence of the IL-I in the oxygen concentrations of $0-100 \%$. B: Stern-Volmer plot $\mathrm{Io} / \mathrm{I}=$ $\mathrm{f}\left(\mathrm{pO}_{2}\right)$ of $\mathrm{H}_{2}$ TPP for $\mathrm{O}_{2}$ sensing.

Figure 5. A: The emission spectra of $\mathrm{H}_{2}$ TPP dye doped in poly(TMSP) in the presence of the IL-II in the oxygen concentrations of $0-100 \%$. B: Stern-Volmer plot $\mathrm{Io} / \mathrm{I}=\mathrm{f}\left(\mathrm{pO}_{2}\right)$ of $\mathrm{H}_{2} \mathrm{TPP}$ for $\mathrm{O}_{2}$ sensing.

The gas concentration giving a signal equal to average of the blank $(n=20)$ plus three times of the standard deviation was reported as the LOD. The detection limits for the $\mathrm{C} 1$ and $\mathrm{C} 3$, were found to be $1.32 \times 10^{-3}$ and $4.12 \times 10^{-4}$, respectively (See Table 3 ). 

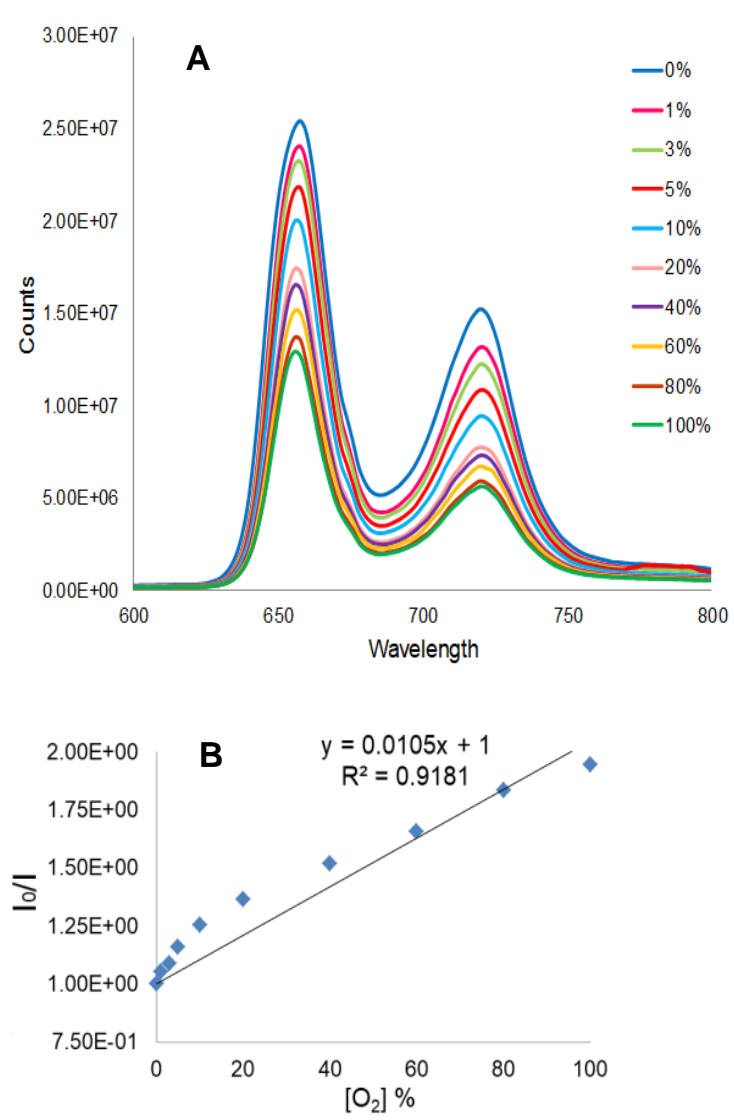

Figure 6. A: The emission spectra of $\mathrm{H}_{2} \mathrm{TPP}$ dye doped in poly(TMSP) in the presence of the IL-III in the oxygen concentrations of 0-100\%. B: Stern-Volmer plot $\mathrm{Io} / \mathrm{I}=\mathrm{f}\left(\mathrm{pO}_{2}\right)$ of $\mathrm{H}_{2} \mathrm{TPP}$ for $\mathrm{O}_{2}$ sensing.

\subsection{Interaction of the AgNPs with the sensing dye}

The oxygen sensing ability of the $\mathrm{H}_{2} \mathrm{TPP}$ dye improved distinctly when the dye molecules had been in close proximity with the AgNPs. The steady-state and time- domain fluorescence based measurements of the lone AgNPs and the $\mathrm{H}_{2}$ TPP dye in the matrix can be helpful to understand the mechanism of the interaction between the dye and AgNPs. Fig. 7 shows the normalized steady-state excitation and emission spectra of the AgNPs and the porphyrin dye in the poly(TMSP) matrix, respectively. AgNPs exhibit a strong emission band at $450 \mathrm{~nm}$ when excited at $360 \mathrm{~nm}$. According to Fig. 7, the emission band of the AgNPs completely overlaps with the excitation band of the $\mathrm{H}_{2} \mathrm{TPP}$ when excited at the $420 \mathrm{~nm}$. It means there is an energy transfer from the AgNPs to the $\mathrm{H}_{2} \mathrm{TPP}$. However, there is a limited overlap between the excitation band of the AgNPs and the excitation band of the $\mathrm{H}_{2} \mathrm{TPP}$. These features make the AgNPs significant potential energy transmitter agents.

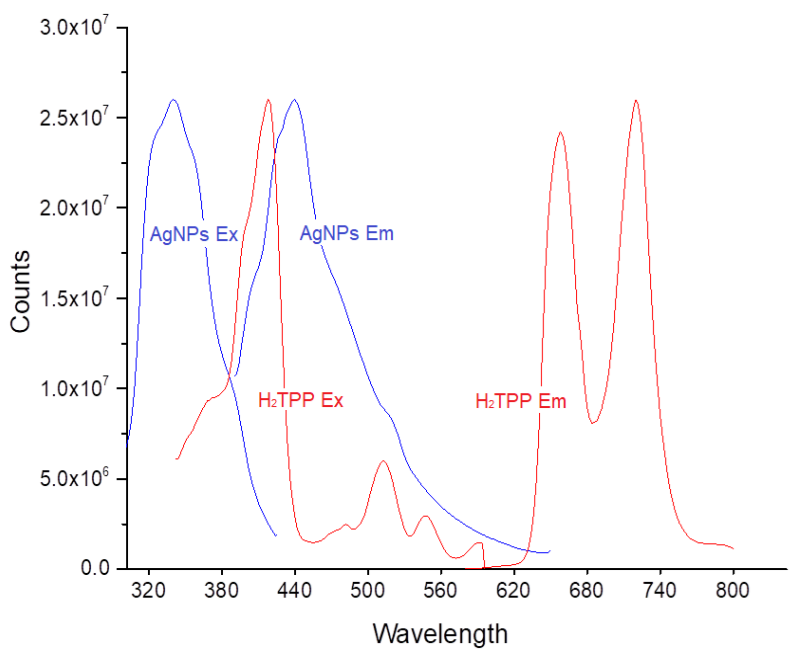

Figure 7. The normalized steady-state excitation and emission spectra of the AgNPs and the $\mathrm{H}_{2}$ TPP dye in the poly(TMSP)-based thin films.

Table 4. Florescence decay-times of the $\mathrm{H}_{2} \mathrm{TPP}$ dye in poly(TMSP)-based thin films in the presence and absence of the IL-II.

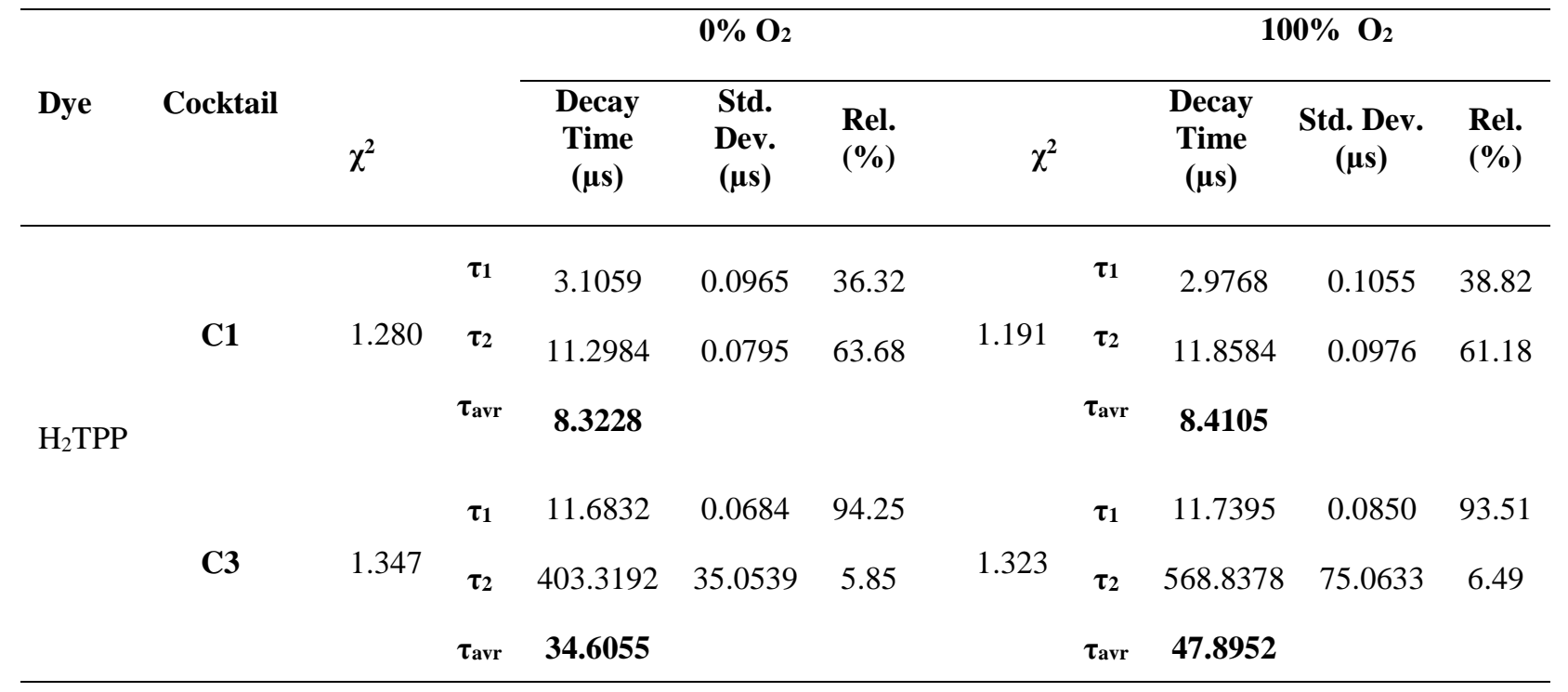


The energy transfer between the donor (silver nanoparticles) and the acceptor $\left(\mathrm{H}_{2} \mathrm{TPP}\right)$ is a function of the concentration of AgNPs. If the number of the AgNPs isn't well optimized the free-phthalocyanine dye becomes self-quenched or the enhancement in the signal cannot be observed. In case of the excess amount of the AgNPs, the interaction between the fluorophore and the silver nanoparticles results with the self-quenching. Therefore, the amount of the additional AgNPs in the polymeric matrix was adjusted as $3.8 \mathrm{mmol} \mathrm{Ag} / \mathrm{kg}$ polymer [18] (See Table 3).

\subsection{Decay-time measurements of the $\mathrm{H}_{2}$ TPP embedded in the polymer matrix}

For comparison, decay curves of the sensing slides were recorded in oxygen-free and fully-oxygenated moieties, respectively. Herein we observed bi-exponential decays for all of the composites, both in oxygenated and deoxygenated conditions. The $\mathrm{C} 1$ and $\mathrm{C} 3$ exhibited luminescence decay times extending to the microsecond region. Short and long decay-time components of the decays and their distribution results for the $\mathrm{C} 1$ and $\mathrm{C} 3$ were shown in the Table 4 . The average decay times of 8.3 and $8.4 \mu$ s were recorded for the oxygen-free and fully-oxygenated conditions for the $\mathrm{C} 1$. However, the C3 exhibited 34.6 and $47.9 \mu$ s decay times for the oxygen-free and fully-oxygenated moieties, respectively. In the absence of the oxygen, we measured decay-time of the metal-free $\mathrm{H}_{2} \mathrm{TPP}$ and the AgNPs/ $\mathrm{H}_{2}$ TPP pair as 8.3 and $34.6 \mu \mathrm{s}$, respectively. Similarly, in the fully oxygenated atmosphere the decay-times for the $\mathrm{C} 1$ and $\mathrm{C} 3$ were measured as 8.4 and $47.9 \mu \mathrm{s}$, respectively (See Table 4). It is known that luminophores with longer $\tau_{0}$ lifetimes are known to have higher oxygen sensitivities and thus are preferred for oxygen sensor studies. The C3, in which contained IL-II additive, exhibited higher $\tau_{0}$ value of $47.9 \mu$ s, when compared to the $\mathrm{C} 1$ that had no additive. This shows the C3 has higher oxygen sensitivity and that is advantageous for the sensor studies.

The recorded decay times can be concluded as an evidence of the presence of fluorescence resonance energy transfer between $\mathrm{H}_{2} \mathrm{TPP}$ and AgNPs which enhances the initial signal intensity of the sensing material prior to the oxygen-induced studies. According to the literature information, the decay-time of the acceptor will exhibit an increase in the presence of the donor because of the FRET from the donor, for this reason, the $\mathrm{H}_{2} \mathrm{TPP}$ dye showed an increase in decay times when doped together with AgNPs with respect to the Ag-free ones.

Decay-time measurements may provide us significant information about on the mechanism of interaction between the $\mathrm{H}_{2}$ TPP dye and the AgNPs. If the quenching is only dynamic, both the emission intensity and the lifetime are affected by the concentration of the triplet oxygen [16]. When the Stern-Volmer plots and lifetime measurements were evaluated together, it can be concluded as the evidence of the dynamic quenching for all of the moieties except that of $\mathrm{C} 1$; which is IL and AgNPs-free.

\subsection{Response and reproducibility performance of the sensor slides}

The time and concentration dependent dynamics of the oxygen sensitive films were also tested and evaluated in terms of reproducibility, reversibility and response time. Fig. 8 reveals oxygen-induced kinetic measurements and reversibility performance tests of the thin film of $\mathrm{C} 3$ ongoing from $100 \% \mathrm{~N}_{2}$ to $100 \% \mathrm{O}_{2}$.

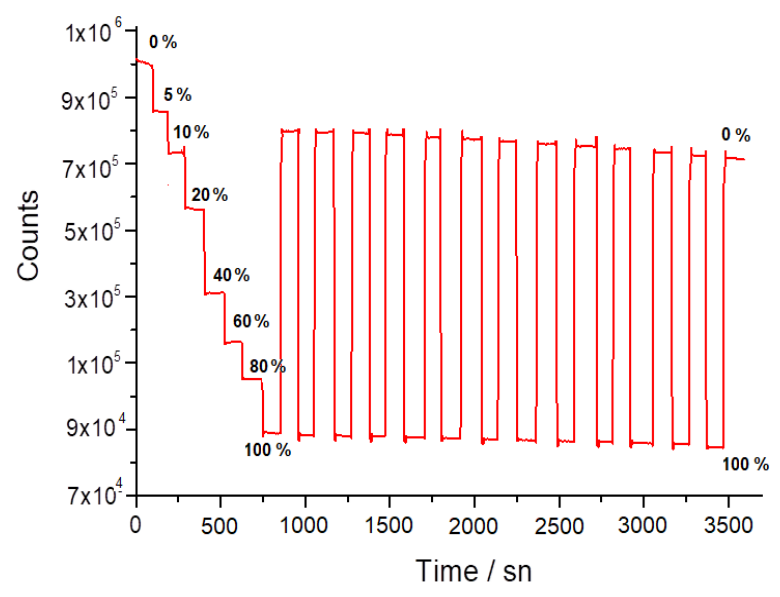

Figure 8. Oxygen-induced emission-based kinetic response of $\mathrm{C} 3$ embedded in poly(TMSP)-based thin film in an alternating atmosphere of oxygen between $0.0-100.0 \%$.

The response time for the $\mathrm{C} 3$, which is the time taken to attain $90 \%$ of the signal intensity when the gas is changed, was found to be 90 seconds. The average recovery time is 150 seconds. The SD of the upper and lower signal intensities was found to be less than $5.0 \%$ for the 12 replicate measurements. From these results, it can be concluded that initial signal intensities of the oxygen sensing slides can easily be recovered and oxygen sensing properties of the $\mathrm{C} 3$ embedded in poly(TMSP)-based thin film is stable and reproducible. The response time of the $\mathrm{C} 1$ was also found to be in the same range of C3 (See Fig. S5).

\section{Conclusion}

In this work, we tested oxygen sensitivity of the commercially available meso-tetraphenylporphyrin in the polymeric matrix along with the AgNPs and observed enhanced sensitivity for the oxygen with respect to the previous reports. Emission and decay-time related data of the metal-free porphyrin dye were acquired in the presence of various types of ILs form of poly(TMSP)based-thin films. In the optimum composition, we used the specified amount of PFC and AgNPs along with imidazolium-based ionic liquids and therefore provided better sensitivity. Additionally, we observed better short and long-term photostabilities. Along with the offered additives, the $\mathrm{H}_{2} \mathrm{TPP}$ dye 
exhibited many advances such as higher oxygen sensitivity more linear calibration plots for larger concentration ranges, and better sensor dynamics. The sensor composites exhibited stable response at least for two months. Our long-term stability studies are still ongoing.

\section{Ethics}

There are no ethical issues after the publication of this manuscript.

\section{References}

1. Potyrailo, RA, Hieftje, GM, Oxygen detection by fluorescence quenching of tetraphenylporphyrin immobilized in the original cladding of an optical fiber, Analytica Chimica Acta, 1998; 370, $1-8$.

2. Bussetti, G, Campione, M, Sassella, A, Duò, L, Optical and morphological properties of ultra-thin $\mathrm{H}_{2} \mathrm{TPP}, \mathrm{H}_{4} \mathrm{TPP}$ and ZnTPP films, Physica Status Solidi (B), 2015, 252(1), 100-104.

3. Weegen, R, Abraham, JP, Meijer, EW, Directing the selfassembly behaviour of porphyrin-based supramolecular systems, Chemistry - A European Journal, 2017, 23, 3773 -3783.

4. Hasselman, GM, Watson, DF, Stromberg JR, Bocian, DF, Holten, D, Lindsey, JS, Meyer, GJ, Theoretical solar-to-electrical energy-conversion efficiencies of perylene-porphyrin lightharvesting arrays, The Journal of Physical Chemistry B, 2006, 110 (50), 25430-25440.

5. Yang, X, Peng, L, Yuan, L, Teng, P, Tian, F, Li, L, Luo, S Oxygen gas optrode based on microstructured polymer optical fiber segment, Optics Communications, 2011, 284, 3462-3466.

6. Mosinger, J, Lang, K, Plistil, L, Jesenska, S, Hostomsky, J, Zelinger, Z, Kubat, P, Fluorescent polyurethane nanofabrics: A source of singlet oxygen and oxygen sensing, Langmuir, 2010, $26,10050-10056$.

7. Topal, SZ, Onal, E, Ertekin, K, Oter, O, Gürek AG, Hirel C, Significant sensitivity and stability enhancement of tetraphenylporphyrin-based optical oxygen sensing material in presence of perfluorochemicals, Journal of Porphyrins and Phthalocyanines, 2013, 17(6), 431-439.

8. Onal, E, Ay, Z, Yel, Z, Ertekin, K Gürek AG, Topal, SZ, Hirel C, Design of oxygen sensing nanomaterial: Synthesis, encapsulation of phenylacetylide substituted $\mathrm{Pd}(\mathrm{II})$ and $\mathrm{Pt}(\mathrm{II})$ mesotetraphenylporphyrins into poly(1- trimethylsilyl-1-propyne) nanofibers and influence of silver nanoparticles, Royal Society of Chemistry Advances, 2016, 6, 9967-9977.

9. Kotiaho, A, Lahtinen, RM, Tkachenko, NV, Efimov, A, Kira, A Imahori, H, Lemmetyinen, H, Gold nanoparticle enhanced charge transfer in thin film assemblies of porphyrin-fullerene dyads. Langmuir, 2007, 23, 13117-13125.

10. Oter, O, Sabanc1, G, Ertekin, $\mathrm{K}$, Enhanced $\mathrm{CO}_{2}$ sensing with ionic liquid modified electrospun nanofibers: Effect of ionic liquid type, Sensor Letters, 2013, 11(9), 1591-1599.

11. Solomon, SD, Bahadory, M, Jeyarajasingam, AV, Rutkowsky, SA, Boritz C, Mulfinger, L, Synthesis and study of silver nanoparticles, Journal of Chemical Education, 2007, 84(2), 322 325 .

12. Lowe, KC, Perfluorochemical respiratory gas carriers: Benefits to cell culture systems, Journal of Fluorine Chemistry, 2002, 118, 19-26.
13. Topal, SZ, Ongun, MZ, Onal, E, Ertekin, $\mathrm{K}$ Hirel, $\mathrm{C}$, Hyperporphyrin effect on oxygen sensitivity of free mesotetraphenylporphyrins, Dyes and Pigments, 2017, 144, 102-109.

14. Anthony, JL, Maginn, EJ, Brennecke, JFJ, Solubilities and thermodynamic properties of gases in the ionic liquid 1-n-butyl-3methylimidazolium hexafluorophosphate, The Journal of Physical Chemistry B, 2002, 106, 7315-7320.

15. Ongun, MZ, Oter, O, Sabanc1, G, Ertekin, K, Celik, E, Enhanced stability of ruthenium complex in ionic liquid doped electrospun fibers, Sensors and Actuators B, 2013, 183, 11-19.

16. Lakowicz, J.R, Principles of Fluorescence Spectroscopy; University of Maryland School of Medicine Baltimore, Press: Springer,Third Edition Maryland, USA, 2006.

17. MacCraith, BD, McDonagh, CM, O’Keeffe, G, Keyes, ET, Vos, JG, O'Kelly, B, McGlip, JF, Fibre optic oxygen sensor based on fluorescence quenching of evanescent-wave excited ruthenium complexes in sol-gel derived porous coatings, Analyst, 1993, 118, 385-388.

18. Ozturk, O, Oter, O, Yildirim, S, Subasi, E, Ertekin, K, Celik, E, Temel, H, Tuning oxygen sensitivity of ruthenium complex exploiting silver nanoparticles, Journal of Luminescence, 2014, $155,191-197$ 